\title{
Putting together the pieces of the puzzle - Part II - Assessing harbour porpoise (Phocoena phocoena) carcasses potentially subjected to grey seal (Halichoerus grypus) predation
}

Abbo van Neer ( $\sim$ abbo.van.neer@tiho-hannover.de )

Stiftung Tierarztliche Hochschule Hannover https://orcid.org/0000-0002-3519-0356

\section{Stephanie Gross}

Tierarztliche Hochschule Hannover

\section{Tina Kesselring}

Tierarztliche Hochschule Hannover

Miguel L. Grilo

Universidade de Lisboa

\section{Eva Ludes-Wehrmeister}

Tierarztliche Hochschule Hannover

\section{Giulia Roncon}

University of Tasmania

Ursula Siebert

Tierarztliche Hochschule Hannover

\section{Research}

Keywords: grey seal, predation, harbour porpoise, lesion, wound pattern, marine mammal, pathology, decision tree, lethal interaction, North Sea

Posted Date: March 25th, 2020

DOI: https://doi.org/10.21203/rs.3.rs-16196/v2

License: (c) (i) This work is licensed under a Creative Commons Attribution 4.0 International License. Read Full License 
The authors have withdrawn this preprint from Research Square 